\title{
¿Castañar para Fruto o para Madera? Consideraciones Sobre su Autoecología
}

\author{
A. Rubio Sánchez \\ Dpto. Silvopascicultura \\ Universidad Politécnica de Madrid \\ E-28040 Madrid \\ Spain
}

\section{LA AUTOECOLOGÍA PARAMÉTRICA DE ESPECIES FORESTALES EN ESPAÑA}

Habitualmente el castaño en España ha sido descrito como una especie que crece en suelos de ladera, profundos, frescos o húmedos, y preferentemente ácidos, con climas suaves sin sequía acusada. Características autoecológicas muy poco precisas que dificultaban de manera notable la tarea de los técnicos forestales, ya que no disponen de información precisa con la que poder valorar la idoneidad de un territorio para el crecimiento de esta especie. Por fortuna, los estudios autoecológicos iniciados en los años 60 por el profesor Gandullo en la E.T.S.I. de Montes de la Universidad Politécnica de Madrid y en el CIFOR del INIA que se centraron en las principales especies del género Pinus de España, a partir de los años 80 comienzan también a desarrollarse con otras especies forestales, especies de hoja caduca como robles, hayas, castaños. Arranca asi una larga y fructífera serie de trabajos que aportan información precisa y cuantificada sobre los principales rasgos autoecológicos del castaño, primero en Asturias y Cantabria (Gandullo et al., 1983) y posteriormente en Extremadura (Rubio, 1993, 1997, Rubio y Gandullo, 1993, 1994; Rubio et al., 1997, 1998), en Navarra (Blanco y Rubio, 1996; Blanco et al., 1997; Rubio et al., 1997), en Galicia (Blanco et al., 2000; Rubio et al., 2001), Cataluña (Rubio et al., 1999, 2002a), Andalucía (Gómez et al., 2001,2002 ) y zona centro de España (Rubio et al., 2002b). La atención que el profesor Gandullo siempre nos transmitió sobre la importancia de hacer llegar los resultados de estos trabajos a los posibles usuarios de los mismos, nos animó a culminar junto a él todos estos trabajos en una monografia publicada por el INIA titulada Las Estaciones Ecológicas de los Castañares Españoles (Gandullo et al., 2004).

El estudio autoecológico desarrollado con el castaño, al igual que los demás estudios autoecológicos paramétricos de otras especies forestales, se han centrado en la consecución de los siguientes objetivos generales: $1^{\circ}$ Definición y clasificación paramétrica de los hábitats de la especie en su área de distribución. $2^{\circ}$ Elaboración de modelos de estimación de la calidad de la estación para la especie, en función de los parámetros ambientales más significativos. $3^{\circ}$ Identificación y cartografia de las áreas potenciales de expansión de la especie. Estos objetivos se han complementado con otros en los que se analizan y tipifican diferentes elementos de los ecosistemas estudiados como el análisis de la vegetación acompañante, la clasificación a partir de índices climáticos, o las clasificaciones edáficas.

\section{METODOLOGÍA}

La metodología empleada para la consecución de los objetivos reseñados anteriormente se puede sintetizar en las siguientes fases

\section{Fase 1: Estratificación del Territorio}

Se pretendía conseguir un diseño objetivo del muestreo, que se realizó de forma independiente para cada estrato. El apoyo fundamental lo hemos encontrado en la clasificación territorial de España (Elena et al., 1997), obtenida a partir de distintos datos del medio físico. Los estratos se establecen fundamentalmente en base a las clases territoriales definidas en la clasificación junto con la información relativa a las diferentes densidades de las masas existentes y su situación espacial en el territorio. 


\section{Fase 2: Muestreo y Toma de Datos de las Parcelas}

Fijados los estratos y diseñado el muestreo de campo, éste se efectuó estableciendo un conjunto de parcelas en cuya prospección se recogieron una serie de datos relacionados con los siguientes aspectos

- Datos fisiográficos, el posicionamiento de la parcela, la altitud del centro de la misma, su pendiente media, su orientación y la pedregosidad superficial existente

- Datos edáficos correspondientes a la descripción y muestreo de un perfil de suelo en el centro de la parcela. Para ello se procedió a la apertura de una calicata, diferenciando los distintos, horizontes edáficos existentes, anotando su espesor, color, grado de presencia de raíces, pedregosidad no muestreable, tránsito al horizonte subyacente, estructura, etc. Se tomaron muestras de tierra de cada horizonte y se enviaron al laboratorio para la realización de los correspondientes análisis edafológicos.

- Datos florísticos correspondientes a la realización de inventarios botánicos en cada parcela.

- Datos selvícolas que atienden a aquellas mediciones realizadas en las parcelas con vistas a la obtención de parámetros caracterizadores de la forma de masa, espesura, etc., así como para la búsqueda de índices de calidad de estación.

\section{Fase 3: Toma de Datos Climáticos}

En relación con la necesidad de efectuar una asignación de valores climáticos a cada una de las parcelas de muestreo, conviene señalar la dificultad que supone el escaso número de observatorios meteorológicos situados en áreas forestales. Por ello algunos miembros del grupo de investigación de autoecología de especies forestales ha desarrollado una serie de modelos de estimaciones termopluvométricas para la España peninsular (Sánchez Palomares et al., 1999), que son del tipo regresión múltiple en función de la altitud, de la posición geográfica (coordenadas $\mathrm{x}$-utm e y-utm) y de la cuenca o subcuenca hidrográfica a que pertenece el punto considerado.

\section{Fase 4: Elaboración de Parámetros Ecológicos}

La siguiente fase es la elaboración de parámetros ecológicos, entendiendo como tales aquellas relaciones numéricas que tratan de cuantificar la influencia que los distintos factores ecológicos del medio ejercen sobre la especie cuya autoecología se pretende estudiar, en este caso el castaño. Se elaboraron los siguientes parámetros (para detalles metodológicos consultar Gandullo et al., 2004):

1. Parámetros Fisiográficos. Cuantifican las condiciones fisiográficas de posición de cada parcela en su entorno geográfico, así como las propias características de las mismas. Se definieron los siguientes parámetros: altitud, pendiente, pedregosidad superficial, insolación, complejidad del entorno, coeficiente de resguardo de vientos. En algún otro estudio hemos ensayado con otros parámetros como insolación general, evaluado para el conjunto de la ladera sobre la que se asienta la parcela, o el parámetro termotopográfico

2. Parámetros Climáticos. Su elaboración parte de los datos climáticos asignados previamente. Estos parámetros se pueden agrupar de la forma siguiente:

1) Evaluadores del régimen pluviométrico: precipitación total anual, precipitación de primavera, precipitación de verano, precipitación de otoño y precipitación de invierno.

2) Evaluadores del régimen térmico: temperatura media anual, temperatura media de las máximas del mes más cálido, temperatura media de la mínimas del mes más frío, oscilación térmica y suma de las doce evapotranspiraciones potenciales mensuales

3 ) Evaluadores del régimen hídrico: suma de superávits, suma de déficits, índice hídrico anual, duración de la sequía e intensidad de la sequía. En algún otro estudio hemos manejado otros índices, evaluadores de la aridez estival, como el índice de Vernet.

3. Parámetros Edáficos. Elaborados a partir de los resultados analíticos obtenidos de las muestras de suelo tomadas en las parcelas, así como de los datos procedentes de la descripción de los perfiles estudiados. Agrupados en tres bloques, se consideran los siguientes:

1) Evaluadores de las propiedades físicas de los suelos: tierra fina, arena, limo, arcilla, 
coeficiente de capacidad de cementación, coeficiente de impermeabilidad debida al limo, humedad equivalente, permeabilidad y capacidad de retención de agua.

2) Evaluadores de las propiedades químicas y de la fertilidad de los suelos: materia orgánica, acidez actual, acidez de cambio, nitrógeno, relación carbono/nitrógeno, carbonato cálcico activo, carbonato cálcico inactivo, fósforo y potasio.

3) Parámetros edafoclimáticos: evapotranspiración real máxima posible en el conjunto del año, sequía fisiológica y drenaje calculado del suelo.

4. Parámetros de la Fitocenosis. A fin de evaluar de manera cuantificada la respuesta biológica se han elaborado una serie de parámetros deducibles, por una parte, de las características morfológicas y selvícolas de las masas en cada parcela y por otra de la propia composición florística.

\section{Fase 5: Definición de Hábitats}

Una vez elaborados estos parámetros se examinó su variabilidad, a fin de establecer los posibles límites de aptitud ecológica de los biótopos que pueden servir de asiento a las masas de la especie estudiada, de acuerdo con el siguiente esquema metodológico: Con los valores de los parámetros establecimos los límites inferior y superior de variación (LI, LS) como el mínimo absoluto y el máximo absoluto para cada parámetro. Situamos los umbrales inferior y superior (UI, US) excluyendo el $10 \%$ de los puntos en los que el parámetro toma los valores menores y otro $10 \%$ excluyendo los valores mayores. A partir de ellos, para cada parámetro definimos el tramo central (intervalo entre UI y US) y los tramos marginales (intervalo entre LI y UI junto con el intervalo entre US y LS). Para el conjunto de todos los parámetros considerados establecimos como hábitats óptimos o centrales aquellos donde los parámetros se encuentran dentro de los tramos centrales. Cuando algunos parámetros se situaron en los tramos marginales se consideraron como hábitats marginales. $\mathrm{Si}$ alguno de los parámetros se situó fuera de los límites establecidos por los valores del intervalo (LI, LS), correspondía a hábitats extramarginales.

Para no extendernos excesivamente nos vamos a detener aquí, sin llegar a comentar las siguientes fases donde los trabajos con otras especies forestales han encontrado gran aplicabilidad al desarrollar los Modelos predictivos de la calidad (Fase 6) y al haber realizado la Identificación y cartografía de áreas potenciales de expansión de la especie (Fase 7).

\section{AUTOECOLOGÍA DEL CASTAÑO (FRUTERO Y MADERERO) EN ESPAÑA}

Así, sin pretender ser demasiado exhaustivo, el estudio realizado con los castañares en España nos ha permitido observar que, desde el punto de vista fisiográfico éstos se localizan desde los $50 \mathrm{~m}$ hasta casi $\operatorname{los} 1500 \mathrm{~m}$ de altitud, aunque principalmente se sitúan entre $\operatorname{los} 450$ y $\operatorname{los} 1000 \mathrm{~m}$. Atendiendo a los valores medios, el clima típico de los castañares españoles es: Mesotérmico, húmedo, nemoral, de inviernos, primaveras y otoños muy húmedos, veranos secos, templado con inviernos frescos y veranos calurosos, continental, con grandes superávits, pocos déficits y con una subsequía prácticamente nula. Desde un punto de vista edáfico podemos destacar por ejemplo que la gran mayoría de los castañares poseen suelos con textura franca, franco-limosa o franco-arenosa, faltando los suelos arcillosos, limosos y arenosos. Así mismo, la práctica totalidad de los castañares españoles viven sobre suelos con $\mathrm{pH}$ medio comprendido entre 4,0 y 6,5 .

El castaño, como especie gestionada desde tiempos inmemoriales por el hombre, se han explotado con dos finalidades bien distintas: para producir fruto -en masas de monte alto y, generalmente, con espesura defectiva-, o para producir madera-normalmente en monte bajo, a turno relativamente corto y en masas de mayor espesura-. Ante esta dualidad las preguntas surgen de manera inmediata: ¿las características autoecológicas del castaño productor de fruto son iguales a las del castaño productor de madera?, ¿es que el hombre, por experiencia secular, ha sabido encontrar las más adecuadas localidades para uno u otro tipo de explotación?, o por el contrario, ¿el destino del castañar está ligado a la naturaleza de la propiedad (pública o privada), o a la mayor o menor demanda de un tipo u otro de 
productos?

Para ello, vamos a intentar aclarar esta cuestión apoyándonos en la metodología anteriormente descrita, analizando si los parámetros estudiados presentan los mismos valores en aquellos castañares destinado a su aprovechamiento para fruto o para madera. El trabajo autoecológico planteado inicialmente no estaba orientado a tal efecto, pero el apoyo que para el diseño del muestreo se hizo en una estratificación territorial ajena al propio trabajo (Elena et al., 1997) es sin duda la responsable de que de las parcelas estudiadas el $49 \%$ estuvieran dedicadas a la producción de fruto y el $51 \%$ a la de madera. La identifícación de tales diferencias se ha realizado mediante un análisis de la varianza, con el que se ha comprobado si el valor de la medias de los parámetros en el grupo de parcelas de uso frutero y en el de uso maderero son o no significativamente diferentes

Así hemos podido comprobar que los castañares fruteros analizados en su conjunto español tienden a encontrarse a mayor cota y en sitios de menor pendiente que los madereros. Igualmente, hemos podido observar que los castañares fruteros se sitúan, con respecto a los madereros, de forma general, bajo clima de mayores precipitaciones invernales, menores precipitaciones estivales, mayor oscilación térmica, mayores suma de superávits hídricos, mayores suma de déficits hídricos y mayor duración e intensidad de la sequía meteorológica. Desde el punto de vista edáfico, los castañares fruteros se encuentran, con respecto a los madereros, en suelos menos arenosos y más limosos, de mayor capacidad de retención de agua, menos ácidos y con menos porcentaje de nitrógeno superfícial. Los castañares fruteros se definen sistemas con menor productividad potencial primaria neta y mayor sequía fisiológica para la vegetación

En definitiva, parece ser que la localización de los castañares productores de fruto españoles tiende a concentrarse en terrenos ondulados de clima mediterráneo húmedo y no costero, con bastante oscilación térmica, fuertes precipitaciones invernales y clara sequía estival que se traduce en abundantes superávits hídricos y fuertes déficits, y, quizás para compensar parcialmente esto último, sobre suelos poco arenosos, bastante limosos, de buena capacidad de retención de agua y no demasiado ácidos. Recíprocamente, parece ser que la localización preferente de los castañares productores de madera son, dentro del hábitat general, los terrenos costeros de cotas bajas y pendientes fuertes con clima de oscilación térmica amortiguada y precipitaciones suficientes en verano para asegurar escasos déficits y casi nula sequía meteorológica aunque las lluvias invernales no sean muy abundantes, y suelos fuertemente ácidos y no excesivamente limosos, sino más bien arenosos, aunque ello implique baja capacidad de retención de agua.

Estas tendencias, que pueden ser matizadas a nivel regional (Rubio y Gandullo, 1993), pueden ser una orientación para cuando se opte por emplear el castaño para reforestar una determinada parcela, se tengan también elementos de juicio sobre la utilización previsiblemente más adecuada bien como productor de fruto o bien de madera. Y para cuestionarse, en ciertos casos, sobre lo adecuado de las transformaciones de castañares de fruto en castañares madereros auspiciadas por algunas administraciones, y la de castañares explotados en monte bajo a monte al to propugnadas desde otros ámbitos.

\section{Citas Bibliográficas}

Blanco, A. and Rubio, A. 1996. Caracterización del hábitat edáfico de los castañares de Navarra. Actas del IV Congreso Nacional de la Ciencia del Suelo. Lérida. España: 333-338

Blanco, A., Rubio, A. and Sánchez Palomares, O. 1997 Caracterización del biotopo de los castañares navarros. Actas I Congreso Forestal Hispano-Luso. Pamplona. España: 213-218

Blanco, A., Rubio, A., Sánchez Palomares, O., Elena, R., Gómez, V. and Graña, D. 2000. Autoecología de los castañares de Galicia (España). Invest. Agrar.: Sist. Recur. For. 9(2):337-361.

Elena, R. (dir) 1997. Clasificación biogeoclimática de España peninsular y balear, M.A.P.A., Madrid.

Gandullo, J.M., Sánchez Palomares, O. and González, S. 1983. Estudio ecológico de las 
tierras altas de Asturias y Cantabria, I.N.I.A., Madrid.

Gandullo, J.M., Rubio, A., Sánchez Palomares, O., Blanco, A., Elena, R. and Gómez, V. 2004. Las estaciones ecológicas de los castañares españoles. Monografias INIA: Serie Forestal. $\mathrm{N}^{\circ}$ 7. Ministerio de Educación y Ciencia. Madrid.

Gómez, V., Sánchez Palomares, O., Blanco, A., Elena, Rubio, A. and Graña, D. 2001. Los suelos de los castañares andaluces. Actas del III Congreso Forestal Español Granada. España: 181-187.

Gómez, V., Blanco, A., Sánchez Palomares, O., Rubio, A., Elena, R and Graña, D. 2002. Autoecología de los castañares andaluces. Invest. Agr.: Sist. Recur. For. 11(1):205226.

Rubio, A. 1993. Caracterización del hábitat edáfico de los castañares extremeños. Actas del Congreso Forestal Español. Pontevedra. España: 423-428

Rubio, A. 1997 Ecología y aprovechamientos de los castañares en Extremadura. Montes, $48: 39-43$.

Rubio, A. and Gandullo, J.M. 1993. Comparación edáfica de los castañares fruteros y madereros extremeños. Actas del XII Congreso Latinoamericano de la Ciencia del Suelo. Salamanca. España: 1759-1767.

Rubio, A. and Gandullo, J.M. 1994. Modelos predictivos de la estructura selvícola en castañares extremeños (España). Ecología, 8:137-150.

Rubio, A., Escudero, A and Gandullo, J.M. 1997a. Sweet chestnut silviculture in an ecological extreme of its range (Extremadura-Spain). Ann. Sci. For. 54(7):667-680.

Rubio, A., Blanco, A. and Sánchez Palomares, O. 1997b. Aportaciones al estudio ecológico de los castañares navarros: suelos, clima y físiografía. Edafología, $3(2): 479-490$.

Rubio, A., Escudero, A. and Gavilán, R.G. 1998 The relationships between floristic and edaphic patterns in managed chestnut stands in Extremadura (W Spain). Ecologie, 29(1-2): 197-200.

Rubio, A., Elena, R., Sánchez Palomares, O., Blanco, A., Sánchez, F. and Gómez, V. 1999. Autoecología de los castañares catalanes. Invest. Agrar.: Sist. Recur. For. 8(2):387-405.

Rubio, A., Elena, R., Sánchez Palomares, O., Blanco, A., Gómez, V. and Graña, D. 2001. Hábitat edáfico de los castañares de Galicia (España). Edafología, 8:1-12.

Rubio, A., Elena, R., Sánchez, O., Blanco, A., Sánchez, F. and Gómez, V. 2002a. Soil evaluation for Castanea sativa afforestation in Norheastern Spain. New Forests $23: 131-141$.

Rubio, A., Sánchez Palomares, O., Gómez, V., Graña, D., Elena, R and Blanco, A. 2002b. Autoecología de los castañares de Castilla (España). Invest. Agr.: Sist. Recur. For. 11(2):373-393.

Sánchez Palomares, O., Sánchez, F. and Carretero Carrero, M.P. 1999. Modelos y cartografía de estimaciones climáticas termopluviométricas para la España Peninsular. M.A.P.A.-I.N.I.A. Madrid. 